\title{
Hereditary Disorders of Dentin: Dentinogenesis Imperfecta Type II and Dentin Dysplasia Type II
}

\author{
Sandhya Shanmugam ${ }^{1}$, Kuzhanchinathan Manigandan ${ }^{2}$, Angambakkam Rajasekaran PradeepKumar ${ }^{3}$
}

\begin{abstract}
Dentin is a mineralized tissue in tooth, produced from odontoblasts, that differentiates from the mesenchymal cells of dental papilla. Hereditary dentin defects are broadly classified into two types, namely, dentinogenesis imperfecta (DGI - type I and II) and dentin dysplasia (DD - type I and II). DGI is an autosomal dominant hereditary disorder, and DD is a rare hereditary disturbance of dentin formation that affects both the primary and the permanent dentition. The purpose of this report was to present a case of DGl-type II and a case of DD-type II to highlight the importance of diagnosing hereditary dentin disorders.

Keywords: Dentin, Dentin discoloration, Dentin disorders, Dentin dysplasia, Dentinogensis imperfecta.

Journal of Operative Dentistry and Endodontics (2020): 10.5005/jp-journals-10047-0091
\end{abstract}

\section{INTRODUCTION}

Dentin is a mineralized tissue forming the the body of a tooth, which serves as a protective covering for the pulp and supports overlying enamel and cementum. Mature dentin is about $70 \%$ mineral, $20 \%$ organic matrix, and $10 \%$ water by weight. Dentin is the product of specialized cells called odontoblasts.'

Hereditary developmental disorders affecting dentin are rare anomalies occurring due to a genetic defect in structural or regulatory proteins in dentin. They include dentinogenesis imperfecta (DGI), dentin dysplasia (DD), regional odontodysplasia, and dentin hypocalcification. ${ }^{2}$

DGI is a hereditary autosomal dominant disorder of dentin formation characterized by opalescent blue to brownish discoloration of teeth. ${ }^{3}$ It can also be associated with osteogenesis imperfecta (OI). ${ }^{4}$ Shields et al. classified DGI into three types: DGI-I, associated with $\mathrm{OI}$ and resulting from mutations in collagen type I genes; DGI-II, which manifests clinically in teeth, similar to DGI-I but in the absence of OI; and DGI-III, a phenotype characterized by large pulp chambers. ${ }^{5}$ In DGI type II, both the primary and the permanent dentitions are affected, whereas in type I the primary dentition is predominantly affected. ${ }^{5}$

DGI-II is one of the most common inherited disorders and affects approximately one person in $8,000 .{ }^{6}$ DGI-II occurs due to mutations in dentin sialophosphoprotein (DSPP) gene. ${ }^{7}$ The color of teeth can vary from brown to blue and is sometimes described as amber or gray. ${ }^{3,5}$ Enamel can show hypoplastic or hypocalcified defects in about one-third of the patients, and it tends to separate from the defective dentin. ${ }^{\text {? }}$

$\mathrm{DD}$ is an autosomal dominant disturbance of dentin formation characterized by normal enamel, atypical dentin formation, and anomalous pulpal morphology. ${ }^{5}$ Witkop in 1972 classified DD into type I: Radicular DD and type II: Coronal DD. ${ }^{3}$

DD type I was illustrated as rootless teeth. It commonly affects the radicular portions of both the primary and the permanent teeth and results in teeth with short roots and mobility with a incidence of 1 in $100,0000^{5,6}$

DD type II is an unusual dentin disorder which is characterized by yellow, brown, or amber-colored translucent primary teeth along with complete pulpal obliteration. The permanent teeth are
1,3 Department of Conservative Dentistry and Endodontics, Thai Moogambigai Dental College and Hospital, Dr M.G.R. Educational and Research Institute, Chennai, Tamil Nadu, India

${ }^{2}$ Department of Conservative Dentistry and Endodontics, Faculty of Dental Sciences, Sri Ramachandra Institute of Higher Education and Research, Chennai, Tamil Nadu, India

Corresponding Author: Angambakkam Rajasekaran PradeepKumar, Department of Conservative Dentistry and Endodontics, Thai Moogambigai Dental College and Hospital, Dr M.G.R. Educational and Research Institute, Chennai, Tamil Nadu, India, Phone: +91 9840135711, e-mail: arpradeependo@gmail.com

How to cite this article: Shanmugam S, Manigandan K, PradeepKumar AR. Hereditary Disorders of Dentin: Dentinogenesis Imperfecta Type II and Dentin Dysplasia Type II. J Oper Dent Endod 2020;5(1):51-55.

Source of support: Nil

Conflict of interest: None

usually seen with normal appearance. Roots are normal in size and shape with a "thistle tube" shaped pulp chamber with denticles. Histologically, teeth with DD are characterized by alterations in dentine (e.g. narrower dentinal tubules, reduced number of tubules, and irregular collagen organization), alterations to the dentino-enamel junction, and increased quantum of rodless enamel compared to healthy teeth. ${ }^{8}$

This article presents two cases, one with DGI type II and another with DD type II.

\section{Case Descriptions}

\section{Case 1}

A 24-year-old female patient reported to a private dental clinic with a chief complaint of gingival bleeding. There was no relevant past medical history. Patient had a previous dental history of orthodontic treatment and orthognathic surgical therapy 4 years ago. Specifically, she had a class III malocclusion and had undergone orthognathic surgical procedure (sagittal split osteotomy) for correction of the same. General examination revealed no abnormalities. On clinical examination, all teeth were yellowish 
to amber brown in color (Fig. 1). There was no clinical evidence of enamel loss and attrition. The orthopantomogram (OPG) (Fig. 2) revealed short roots with pulpal oblitreration and bulbous crowns along with cervical constriction. OPG also revealed a surgical plate on the body of the mandible. The patient was provisionally diagnosed with DGI type II with generalized gingivitis.

\section{Treatment}

Appropriate periodontal treatment was done. Esthetic treatment options including bleaching and veneers were explained to the patient, and the patient was advised to come for regular recall dental visits for monitoring DGI II.

\section{Case 2}

A 10-year-old female patient came to the private dental clinic with a chief complaint of fractured front teeth. There was no relevant past medical history. Patient revealed a past dental history of trauma six months ago with complete crown fracture in upper front tooth region. General examination revealed all vital signs within normal range, with no pallor, icterus, cyanosis, clubbing, or edema. Intraoral examination revealed fracture of crown en masse in 11 and 21 as was evident clinically, and radiographic evaluation revealed root stumps in relation to 11 and 21 (Fig. 3). Other findings include clinically missing 13 and 23 (Fig. 4). Clinical examination also revealed enamel with normal color, texture, and consistency with minimal attrition. There was no pitting or softening of enamel. On pulp sensibility testing 12, 22, and mandibular anteriors responded within limits.

Intraoral periapical radiographs revealed teeth having normal enamel thickness. However, the dentin was found to be very thin. Pulp chambers were enlarged and extending into the roots; however, the root length was normal. Sudden constriction was noticed at the base of the pulp chamber along with very thin, narrow root canals revealing a typical "thistle tube appearance in mandibular anteriors as well as in maxillary and mandibular posteriors" (Figs 5 to 8 ). Since the patient was 10 years old, root formation was not complete in both maxillary and mandibular premolars with the appearance of periapical radiolucency.

When the parents were interviewed, no relevant family history was apparent. Differential diagnoses considered were DGI type I, DD type I, DD type II, and odontodysplasia.

A provisional diagnosis of DD type II-autosomal dominant hereditary disorder-was arrived at due to the radiographic appearance of thistle-tube appearance of pulp and root canal

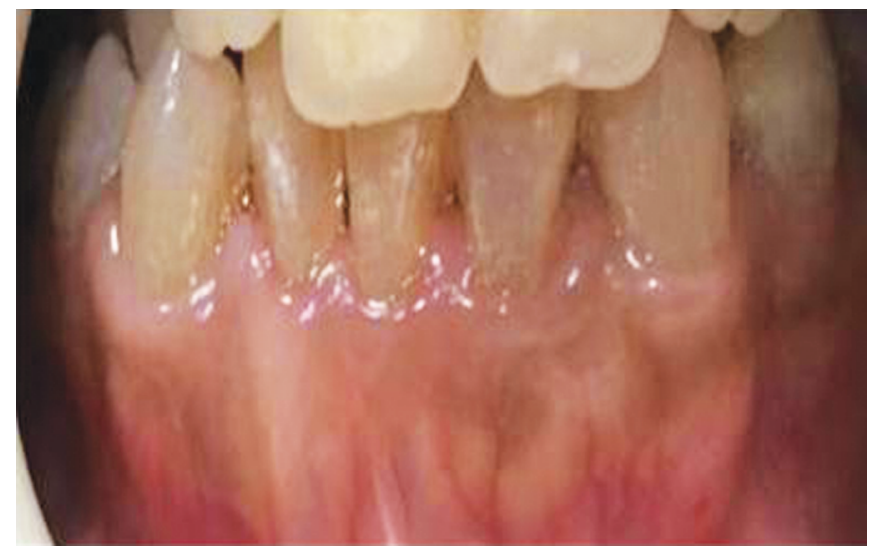

Fig. 1: Dentinogenesis imperfecta type II with yellowish to amber brown discoloration in teeth space calcification in mandibular anterior, mandibular posterior, and maxillary posterior teeth (Figs 5 to 8 ). In this case, maxillary and mandibular premolars were associated with the appearance of periapical radiolucency. Also, root formation was not complete. However, no treatment was done, as the teeth were not carious or otherwise defective. It was decided to observe and wait for complete root formation.

\section{Treatment}

In this case of DD type II, no treatment was done for maxillary or mandibular posterior teeth or existing anterior teeth. However, both 11 and 21 root stumps were planned for extraction followed by replacement. Patient was advised to come for regular recall dental visits for monitoring root formation in maxillary and mandibular posterior teeth and DD type II.

\section{Discussion}

In humans, there are about 20 different types of collagen that have been expressed from 22 different collagen genes. ${ }^{11}$ Dentin primarily consists of type I collagen that constitutes $85 \%$ to $90 \%$ of the organic matrix. ${ }^{12}$ The noncollageneous proteins evident in dentin are DSPP ${ }^{13}$ derivatives, namely dentin sialoprotein (DSP), dentin phospoprotein (DPP), and dentin glycoprotein (DGP). ${ }^{14}$ It has been proved that DSPP is an important factor for dentin formation. ${ }^{6}$

DGI is one of the most commonly occurring hereditary conditions of dentin formation, wherein the dental papilla of either or both the primary and the permanent dentition can be affected. ${ }^{9}$ DGI II is caused by mutation in the DSPP gene (gene map locus 4q21.3), encoding DPP and DSP. ${ }^{15}$

Diagnosis and differential diagnosis is very essential in differentiating the types of DGI. Both DGI-I and DGI-II have similar clinical, radiographical, and histological findings. However, patients with DGI-I can also suffer from OI, which demands a more comprehensive management. In our case, the patient was diagnosed with DGI-II, as no evidence of OI or other types of DGI was found. As DGI-II is a hereditary disease, to confirm our diagnosis, gene sequencing can be done to investigate the implicated DSPP mutation. ${ }^{4}$ Also, we could not identify any relevant family history.

Treatment of DGl is focused on several objectives to maintain the dental health and preserve vitality, form and size of the dentition; to provide esthetic appearance; to rehabilitate with a functional dentition; to prevent loss of vertical dimension; to avoid interfering with eruption of the remaining permanent teeth; and to allow normal growth of the facial bones and temporomandibular joint. ${ }^{16}$ However, in our case, the patient was only treated for her primary complaint. Esthetic treatment modalities were explained

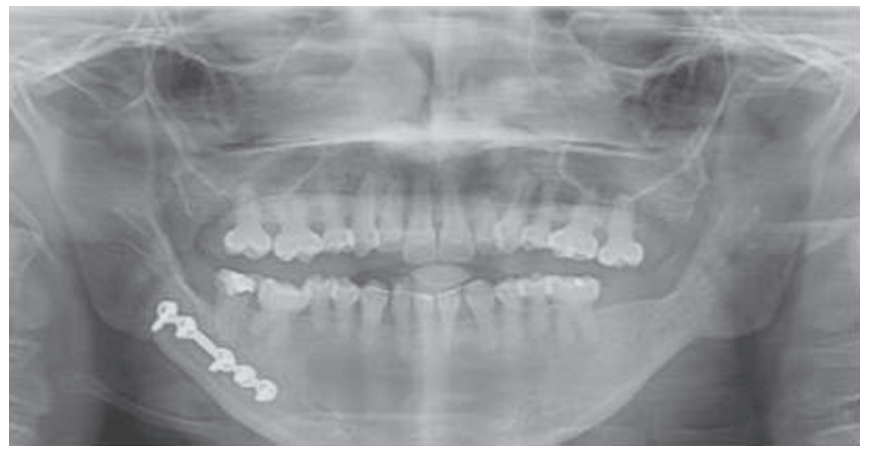

Fig. 2: OPG of case report 1(DGI-II) 

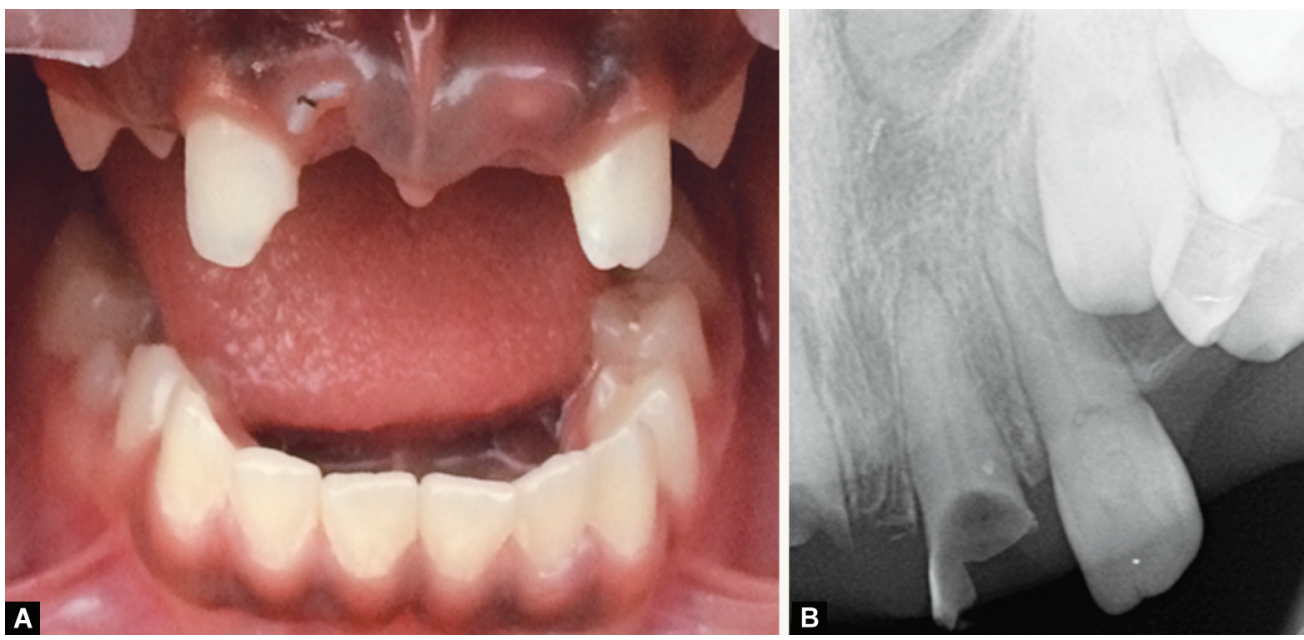

Figs 3A and B: (A) Fracture of crown en masse in 11 and 21; (B) Intraoral periapical radiograph reveals root stumps of 11, 21

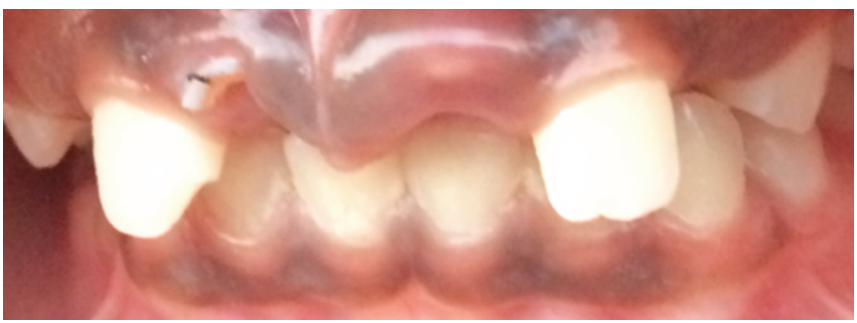

Fig. 4: Missing teeth in relation to upper front teeth region 9,10

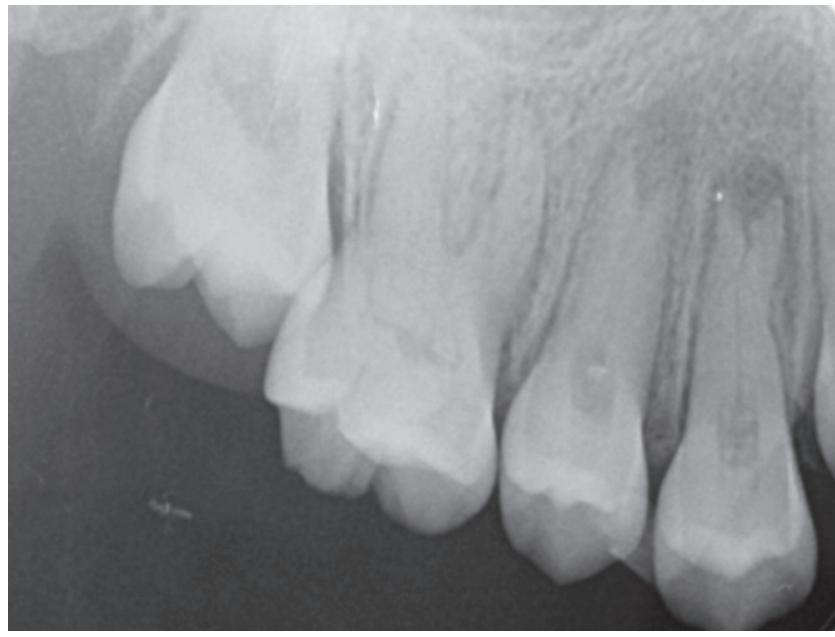

Fig. 6: Intraoral periapical radiograph of maxillary posterior teeth reveals early obliteration of root canals with thistle tube appearance

to the patient. We have educated the patient to report for follow-up dental visits (every 6 months) to monitor the DGI II condition.

DD is a rare genetic disorder of dentin with unknown etiology. Dentin dysplasia (DD) is an autosomal dominant and hereditary disease caused by mutation of DSPP gene. DSPP mutations have been reported to be associated with the pathogenesis of dentin diseases without bone involvement, including DGI-II, DGI-III, and DD-II. ${ }^{17}$ Logan et al. stated that dental papilla is responsible for abnormal root development and that when multiple degenerative foci within the papilla gets calcified, it can lead to reduced growth

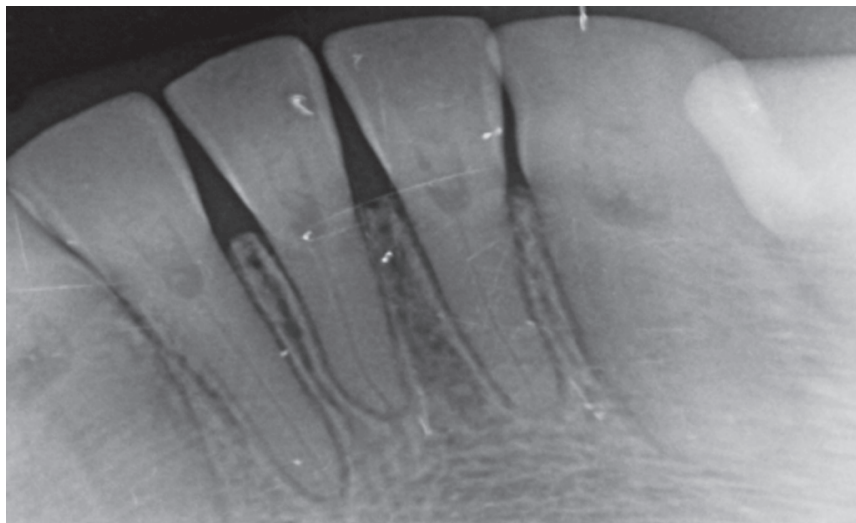

Fig. 5: Intraoral periapical radiograph of mandibular anterior teeth shows early obliteration of root canals with thistle tube appearance

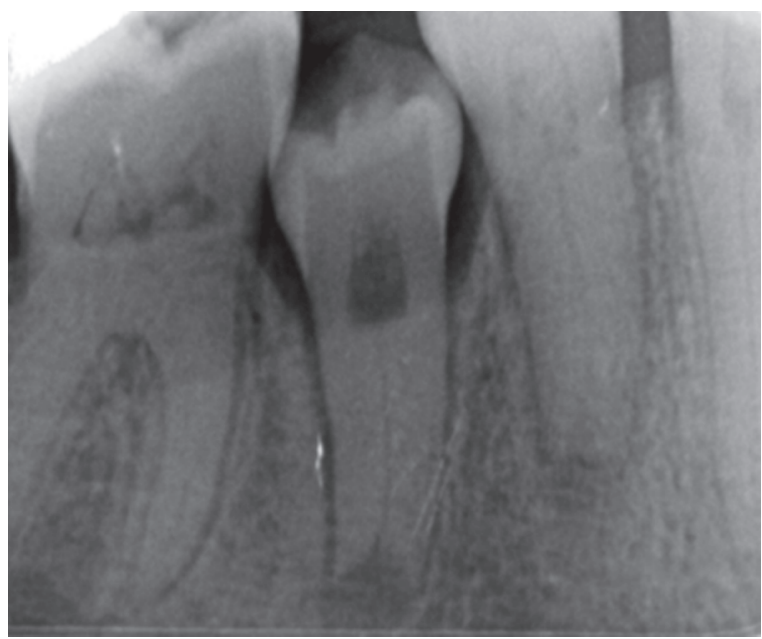

Fig. 7: Intraoral periapical radiograph of mandibular posterior teeth shows early obliteration of root canals with thistle tube appearance

and obliteration of pulp space. ${ }^{18}$ Wesley et al. stated that DD is caused by an interaction of odontoblasts with ameloblasts that lead to abnormal differentiation and/or function of the odontoblasts. ${ }^{19}$ 


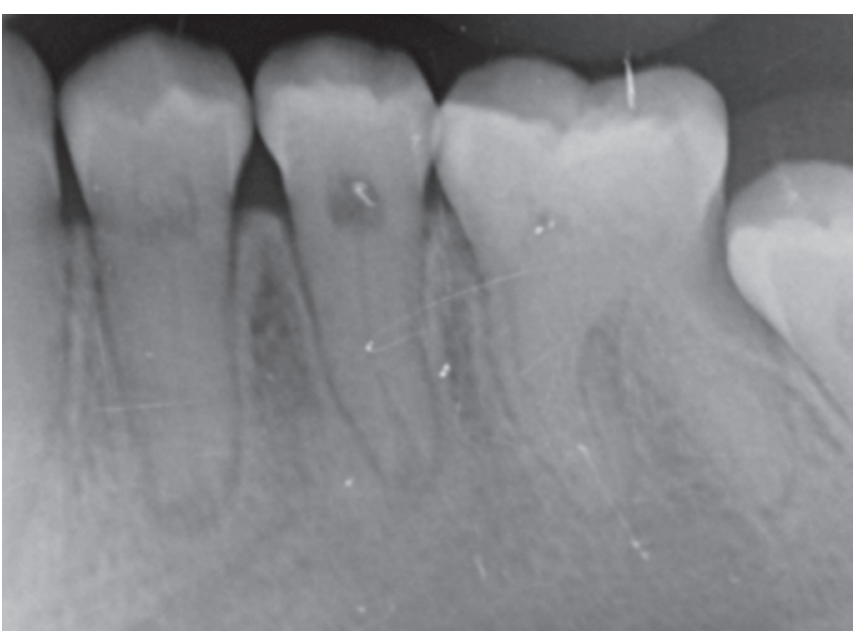

Fig. 8: Intraoral periapical radiograph of mandibular posterior teeth shows early obliteration of root canals with thistle tube appearance

Usually in DD, permanent teeth are normal in color, but the pulp chambers are obliterated by abnormal dentin. ${ }^{7}$ It is very essential to differentiate between DD type I and DD type II. In DD type I, deposition of amorphous dentine or pulp stones leads to obliteration of root canals, ${ }^{5}$ and there may be increased mobility.

In DD type II, the deciduous teeth have features similar to that of DGI-II. In the permanent dentition, the teeth are of normal shape, form, and color, while pulp cavities can have thistle tube deformity and pulp stones. In some cases, features characteristic of DGI-II are observed, such as bulbous crowns with cervical constriction, mild discoloration, and pulp obliteration..$^{5,11}$ In our patient, the clinical crowns were normal in shape with radiological evidence of enlarged pulp chamber extending into roots, which gives "thistle tube appearance", with normal root length which were typical findings of DD type II. ${ }^{20}$ Also, teeth did not exhibit mobility.

DD is usually an autosomal dominant condition, ${ }^{5}$ but in our patient, we could not elicit any associated familial history of DD; thus, our patient in this case report was considered to be a first generation carrier of DD.

Treatment plan for DD is aimed toward preserving the existing teeth, improving occlusion, mastication, and aesthetics for the patient. Regular follow-up and conservative treatment is the primary choice of treatment plan in DD. ${ }^{21}$ Routine endodontic treatment can be done when indicated. In our patient extraction of root stumps (11 and 21) followed by replacement was planned. Esthetic treatment planning was not considered at this point due to young age of the patient.

DGI-II affected teeth are usually discolored and can present with loss of enamel. ${ }^{3}$ Tooth wear seen in primary dentition can be restored with stainless steel crowns to prevent further wear and maintain occlusal dimensions. ${ }^{22}$ Composite resin restorations provide a conservative treatment option for primary teeth ${ }^{23}$ though the longevity is questionable. ${ }^{10}$ Missing/non-restorable teeth due to wear and fracture in adult patients can be treated with prosthodontic rehabilitation and dental implants. ${ }^{24}$ Pulp space ${ }^{5}$ obliteration in permanent teeth can present complications during endodontic treatment. ${ }^{22}$

Patients with DD type II can present with tooth discoloration in the primary dentition which may be treated with composite restorations. However, data on composite bonding to teeth with
DD type II is scarce. Root canal treatment followed by crowns can be recommended for teeth with pulpal necrosis in permanent teeth with DD type I. ${ }^{21}$ When teeth are unsalvageable, removal and reconstruction with implant supported prosthesis can be done. ${ }^{25}$ Patients with DD type II usually do not present with clinical defects in the permanent dentition. Routine follow-up is done to prevent periodontal disease and dental caries in order to retain the teeth as long as possible.

\section{Conclusion}

This case report emphasizes the correct diagnosis of hereditary dentin defects such as DGI type II and dentin dysplasia type II.

\section{References}

1. Nanci A. Dentin-pulp complex. Ten cate's oral histology development, structure and function. 9th ed., Mosby Elsevier; 2017.

2. Gulati N, Juneja S, Singh A, et al. A histological continuum between dentinogenesis imperfecta and dentin dysplasia: a case report with literature review. Indian J Dent Res 2019;30(4):643-646. DOI: 10.4103/ ijdr.IJDR_318_18.

3. Witkop CJ. Hereditary defects in enamel and dentin. Acta Genet Stat Med 1957;7(1):236-239. DOI: 10.1159/000150974.

4. Fan F, Li N, Huang $S$, et al. A multidisciplinary approach to the functional and esthetic rehabilitation of dentinogenesis imperfecta type II: a clinical report. J Prosthet Dent 2019;122(2):95-103. DOI: 10.1016/j.prosdent.2018.10.028.

5. Shields ED, Bixler D, El-Kafrawy AM. A proposed classification for heritable human dentine defects with a description of a new entity. Arch Oral Biol 1973;18(4):543-553. DOI: 10.1016/0003-9969(73) 90075-7.

6. Kim JW, Simmer JP. Hereditary dentin defects. J Dent Res 2007;86(5):392-399. DOI: 10.1177/154405910708600502.

7. Hart PS, Hart TC. Disorders of human dentin. Cells Tissues Organs 2007;186(1):70-77. DOI: 10.1159/000102682.

8. Brenneise CV, Conway KR. Dentin dysplasia, type II: report of 2 new families and review of the literature. Oral Surg Oral Med Oral Pathol Oral Radiol Endod 1999;87(6):752-755. DOI: 10.1016/S10792104(99)70175-7.

9. Sivapathasundharam B. Shafer's Textbook of Oral Pathology. 8th ed., Elsevier; 2016.

10. Gallusi G, Libonati A, Campanella V. SEM-morphology in dentinogenesis imperfecta type II: microscopic anatomy and efficacy of a dentine bonding system. Eur J Paediatr Dent 2006;7(1):9-17.

11. Myllyharju J, Kivirikko KI. Collagens and collagen-related diseases. Ann Med 2001;33(1):7-21. DOI: 10.3109/07853890109002055.

12. Linde A, Bhown M, Butler WT. Noncollagenous proteins of dentin. A re-examination of proteins from rat incisor dentin utilizing techniques to avoid artifacts. J Biol Chem 1980;255(12):5931-5942.

13. MacDougall M, Simmons D, Luan X, et al. Dentin phosphoprotein and dentin sialoprotein are cleavage products expressed from a single transcript coded by a gene on human chromosome 4. J Biolog Chem 1997;272(2):835-842. DOI: 10.1074/jbc.272.2.835

14. Yamakoshi Y. Dentinogenesis and dentin sialophosphoprotein (DSPP). J Oral Biosci 2009;51(3):134-142. DOI: 10.1016/S1349-0079(09) 80021-2.

15. MacDougall M, Jeffords LG, Gu TT, et al. Genetic linkage of the dentinogenesis imperfecta type III locus to chromosome 4q. J Dent Res 1999;78(6):1277-1282. DOI: 10.1177/00220345990780061301.

16. Devaraju D, Devi B, Vasudevan V, et al. Dentinogenesis imperfecta type I: A case report with literature review on nomenclature system. J Oral Maxillofac Pathol 2014;18(4):131-134. DOI: 10.4103/0973029X.141363.

17. Acevedo AC, Santos LJS, Paula LM, et al. Phenotype characterization and DSPP mutational analysis of three Brazilian dentinogenesis 
imperfecta type II families. Cells Tissues Organs 2009;189(1-4): 230-236. DOI: 10.1159/000152917.

18. Logan J, Beck H, Silverman Jr S, et al. Dentinal dysplasia. Oral Surg 1962;15(3):317-333. DOI: 10.1016/0030-4220(62)90113-5.

19. Wesley RK, Wysocki GP, Mintz MS, et al. Dentin dysplasia type I. Clinical, morphologic, and genetic studies of a case. Oral Surg 1976;41(4):516-524. DOI: 10.1016/0030-4220(76)90279-6.

20. Daryani D, Nair G, Naidu G, et al. Dentin dysplasia type II: an exclusive report of two cases in siblings. J Indian Acad Oral Med Radiol 2017;29(2):132-134. DOI: 10.4103/jiaomr.JIAOMR_23_16.

21. Ravanshad S, Khayat A. Endodontic therapy on a dentition exhibiting multiple periapical radiolucencies associated with dentinal dysplasia type 1. Aus Endod J 2006;32(1):40-42. DOI: 10.1111/j.17474477.2006.00008.x.
22. Pettiette MT, Wright JT, Trope M. Dentinogenesis imperfecta: endodontic implications. Case report. Oral Surg Oral Med Oral Pathol Oral Radiol Endod 1998;86(6):733-737. DOI: 10.1016/S10792104(98)90213-X.

23. Beltrame AP, Rosa MM, Noschang RA, et al. Early rehabilitation of incisors with dentinogenesis imperfecta type II - case report. J Clin Pediatr Dent 2017;41(2):112-115. DOI: 10.17796/1053-462841.2.112.

24. Bencharit S, Border MB, Mack CR, et al. Full-mouth rehabilitation for a patient with dentinogenesis imperfecta: a clinical report. J Oral Implantol 2014;40(5):593-600. DOI: 10.1563/AAID-JOI-D-12-00217.

25. Depprich RA, Ommerborn MA, Handschel JG, et al. Dentin dysplasia type I: a challenge for treatment with dental implants. Head Face Med 2007;22(3):31. DOI: 10.1186/1746-160X-3-31. 